\title{
DIE MENSCHENRECHTE IM VERSTANDNIS DES ISLAM
}

\author{
Von Udo Steinbach
}

Ein gemeinsames Verständnis aller Völker über die Menschenrechte ist eine wesentliche Voraussetzung für die Verwirklichung der universalen politischen Ziele, die von den Vereinten Nationen angestrebt werden: der Erhaltung des Friedens, der Zusammenarbeit der Staaten und der Entwicklung bislang zurückgebliebener Völker. In diesem Sinne hat die „Menschenrechtserklärung“ der Vereinten Nationen vom 10. Dezember 1948 ihre weit über das rein Deklamatorische hinausgehende politische Bedeutung.

Die weltweite Anerkennung indessen, die sie gefunden hat, läßt leicht vergessen, daß die „Menschenrechte“ im Verlaufe der europäischen Geschichte der Neuzeit eine spezifische Bedeutung erlangt haben, die untrennbar ist von deren historischem, politischem, religiösem und kulturellem Hintergrund. Der Inhalt der "Menschenrechte" ist bestimmt durch die Auffassung vom Wesen des Menschen, seiner Stellung in der Welt, und der Beziehung von Diesseits und Jenseits, von Staat und Religion, die sich seit der Renaissance herausgebildet hat.

Deshalb liegt es nahe anzunehmen, daß in einem so durchaus eigenen religiöspolitischen Rahmen, wie es der Islam ist, die "Menschenrechte“ einen eigenen Inhalt gewonnen und eine eigene Entwicklung genommen haben; und es ist zu untersuchen, ob nicht selbst in der Gegenwart, nachdem der islamische Raum sich unter dem Eindruck des Zusammenstoßes mit dem Westen seit Beginn des 19. Jahrhunderts religiös wie politisch modernisiert und europäische Strukturen übernommen hat, Spuren der für ihn einst charakteristischen Bestimmung des Menschen und seiner „Rechte" erkennbar sind.

Die folgende Untersuchung wird in drei Teile gegliedert: Zunächst wird die Beziehung zwischen dem System der islamischen Religion, der staatlich-politischen Wirklichkeit und der Konzeption vom Individuum, seinem Wert und seiner Position zwischen Diesseits und Jenseits, analysiert. Es folgt eine Darstellung der Argumente, mit denen islamische Modernisten und Apologeten die Existenz von „Menschenrechten“ im Islam nachzuweisen suchen. Im dritten Teil schließlich wird versucht, Elemente der traditionellen Konzeption in der politischen Gegenwart des islamischen Nahen Ostens aufzuzeigen.

\section{Staat und Individuum im Islam}

Der Islam ist nicht lediglich eine Religion im Sinnne eines theologisch-dogmatischen Systems. Vielmehr impliziert er zugleich die Gründung eines spezifisch islamischen Staates, enthält also untrennbar eine geistlich-religiöse wie weltlich-politische Komponente. Mit der Gründung eines durch den Propheten Muhammad geführten Gemeinwesens in Medina begann die Religion, auch die weltlichen Geschicke der 
Gemeinde zu gestalten. Es ist kein Zufall, daß der Zeitpunkt der Übersiedlung Mohammeds von Mekka nach Medina (622), der „hidschra“, zugleich der Beginn der islamischen Zeitrechnung ist ${ }^{1}$.

Es ist zunächst notwendig, einen Blick auf einige grundlegende Züge zu werfen, die aus der Einheit von Religion und Politik im Islam resultieren und die die Stellung des einzelnen Menschen im Staat und die Beziehungen von Staat und Mensch bestimmen, die also mithin die Rahmenbedingungen für die Definition von "Menschenrechten" darstellen.

Der islamische Staat ist die politische Organisation der Gemeinde der Bekenner der islamischen Religion (umma). Die rechtliche Grundlage des Zusammenlebens der Muslime ist das islamische Gesetz, die „sharî’a“, die die Beziehungen des einzelnen Menschen zu den anderen Menschen, zum Staat und zu Gott regelt. Die "sharî’a“ ist der unmittelbare Ausdruck des Willens Gottes; durch sie lenkt Gott selbst die Geschicke seiner Gemeinde. Gott selbst ist der Gesetzgeber; das Gesetz ist von ihm ein für allemal im Koran gegeben worden. Auch die Exekutive liegt bei dem allmächtigen Gott: Er delegiert sie an einen Mittelsmann, d. h. an den Propheten, und nach dessen Tode an den Imam oder Kalifen, „khalîfat rasûl Allâh“, den Stellvertreter des Gesandten Gottes.

Es wird deutlich, welche überragende Bedeutung in der religiös-politischen Konzeption des Islam dem Staate zukommt. Dieser ist der Träger des göttlichen Willens im Diesseits; er ist das Instrument, durch welches Gott seine Gemeinde über die Erde ausbreitet; durch ihn wird Gottes Wirken über die ganze Erde bestätigt. Der islamische Staat hat das Ziel, eine Gemeinde zu organisieren, in der es einer möglichst großen Anzahl von Menschen (im letzten allen Menschen) möglich ist, moralisch wie physisch in Übereinstimmung mit dem Gesetz Gottes, d. h. Allahs, zu leben ${ }^{2}$.

Zwei Aspekte der Stellung des einzelnen lassen sich daraus ableiten:

- Zum einen: Der Koran betont wieder und wieder die Größe und Gewalt Gottes, seine Allmacht im Universum und gegenüber jedwedem auf Erden. Ihm gegenüber ist der einzelne ein Nichts; Selbständigkeit und Selbstbehauptung Gott gegenüber sind ketzerische Frevel.

- Zum anderen: Angesichts der Aufgabe, die islamische Gemeinde über die ganze Welt auszubreiten und den Anspruch der Universalherrschaft Allahs zu verwirklichen, ist der Wert des einzelnen nicht an sich, sondern durch eine übergeordneteAufgabe bestimmt. Insofern er teilnimmt an der historischen Aufgabe der islamischen Gemeinde, durch ihre Aktivität im Diesseits die Existenz, Allmacht und Einzigkeit Gottes zu bezeugen, gewinnt er die Legitimation für sein Dasein. Nur im religiösen Raum also kommt dem Individuum überhaupt Gewicht zu. Das bedeutet aber auch, daß nicht dem einzelnen Muslim, sondern dem Staat als ganzem zentrale Bedeutung zufällt: Die islamische Ordnung ist als Ganzes werthaft, weil in ihr allein das Walten Gottes zum Ausdruck kommt; und die Aufrechterhaltung dieser Ordnung ist prioritär, da nur in ihrem Rahmen die religiöse und politische Existenz des einzelnen überhaupt möglich ist.

Der Absolutheitsanspruch des islamischen Staatswesens als ganzem über den einzelnen, zunächst verkörpert in der Person des Kalifen, später in der Person eines

1 Vgl. Fritz Steppat: Der Muslim und die Obrigkeit. In: Zeitschrift für Politik, N.F. Bd. XII, 1965, S. $319-332$.

2 S. Muhammad Asad: The Principles of State and Government in Islam, Berkeley and Los Angeles, 1961, S. $30 \mathrm{ff}$. 
jeden beliebigen Herrschers und seiner Administration, ist in der politischen Geschichte der islamischen Welt aber auch in der sie begleitenden "politischen Theorie" immer wieder deutlich geworden. Zwar gilt es zunächst als ausgemacht, daß man der Obrigkeit nur dann gehorcht, wenn ihre Befehle sich im Rahmen der Gesetze halten, vielleicht sogar nur dann, wenn sie nicht bloß im speziellen Fall, sondern generell gottgehorsam ist ${ }^{3}$. Bald aber setzt sich die Ưberzeugung durch, daß einem Herrscher auf jeden Fall zu gehorchen, die staatliche Ordnung auf jeden Fall aufrechtzuerhalten sei. Zahlreich sind die diesbezüglichen Aussprüche, die dem Propheten zugeschrieben werden. So heißt es: „Auch mit einem sündigen Herrscher sichert Gott die Straßen, bekämpft er den Feind im Glaubenskrieg, zieht er die Steuer ein; auch unter einem sündigen Herrscher werden die gesetzlichen Strafen vollzogen, findet die Pilgerfahrt nach Mekka statt, kann der Muslim in Sicherheit bis zu seinem Tode die gottesdienstlichen Handlungen verrichten“4. Oder: „Der Gesandte Gottes sagte: ,Die besten eurer Führer sind diejenigen, die ihr liebt, und die, die euch lieben; diejenigen, über die ihr Segen aussprecht, und diejenigen, die Segen über euch aussprechen. Die schlechtesten eurer Führer sind die, die ihr haßt, und die euch hassen; die, denen ihr flucht, und die euch fluchen'. Wir fragten: ,O, Gesandter Gottes! Sollten wir sie nicht stürzen, wenn letzteres der Fall ist?‘ Und er antwortete: „Nein, solange sie nur das Gebet unter euch aufrechterhalten "“s.

Die Zitate zeigen, daß die Aufrechterhaltung der staatlichen Autorität - weitgehend ohne Rücksicht auf ihre moralische Qualität - oberste Pflicht der Muslime ist. Wer über die faktische Macht verfügt - sofern er nur den islamischen Charakter des Staates nicht zerstört --, ist der anerkannte Herrscher. Die Aufrechterhaltung des Staates als der irdischen Manifestation Gottes hat Priorität vor der Wohlfahrt des einzelnen Bürgers. Dem Staat gegenüber bestehen dem einzelnen keine Rechte, die er jenem gegenüber geltend machen könnte und bei deren Nichtbeachtung er sich gegen ihn erheben könnte.

Daraus folgen ei:-ige für das Verständnis von „Menschenrechten“ im Islam wesentliche Züge des islamischen Staates. Zum einen die Übertragung der rigorosen Einheit Gottes auf die staatliche Ordnung: Dem zentralen Dogma, daß Gottes Wesen eines und umfassend ist, entspricht die Auffassung von der Einheit und Allgewalt des Staates. Hieraus resultiert die Tatsache, daß die islamische Staatslehre keine Instanzen kennt, deren Aufgabe es wäre, die politische Machtausübung im Leben der Gemeinde zu kontrollieren. Alle Gewalten sind von der Macht der Zentralgewalt - im Idealfall dem Kalifen - abgeleitet, die wiederum ihre Machtfülle von Gott erhält.

Aus der Einheitsvorstellung folgt ferner, daß die islamische Staatstheorie niemals die Existenz autonomer menschlicher Institutionen im Inneren der Gemeinde der Gläubigen hat anerkennen wollen. Die Allmacht Gottes, auf Erden auf seinen Statthalter übertragen, duldet nicht die Existenz irgendeiner Gewalt, die aus einer anderen Quelle abgeleitet ist. Ihre Anerkennung auf Erden würde eine Variante von Polytheismus (shirk) bedeuten. Im Grunde ist dies die gleiche Haltung, die auf dem Gebiet der Theologie die Vorstellung zurückweisen läßt, daß Gott Attri-

3 S. Steppat, a. a. O., S. 321; mit Belegen.

4 As-Suyûtî: ad-durr al-manthûr, Kairo A. H. 1314, II, S. 178; vgl. Steppat, a. a. O., S. 325

5 Nach M. Asad, a. a. O., S. 78. 
bute zukommen könnten, die eine eigene Existenz außerhalb des göttlichen Wesens besitzen.

Schließlich ist die "juristische Person“ im Islam unbekannt; daraus folgt der "Ein-Mann-Charakter" etwa von Gerichten und anderen Institutionen. Der Herrscher bestimmt einen Gouverneur, einen Richter oder einen Befehlshaber der Truppen, um einen Teil der Funktionen, die ihm selbst von Gott übertragen sind, ausführen zu lassen. Dagegen ist die Einsetzung eines Gremiums wie eines Rates, eines Kollegiums oder einer Kommission unbekannt. So finden wir keine Gerichte als geschlossene Institutionen, keine Gemeindevertretungen und keine kooperativen Gruppen, die diese oder jene Funktion auf den Gebieten der Rechtsprechung oder Administration auszuüben hätten ${ }^{6}$.

So läßt sich hier zusammenfassend feststellen, daß im Rahmen der islamischen Staatstheorie das Problem der "Menschenrechte" sich nicht so stellen konnte wie in Europa, das seit der Renaissance eine spezifische politische und kulturelle Entwicklung vollzogen hatte und den Menschen als eine Größe mit unveräußerlichen, fundamentalen Rechten, die ihm qua Menschen zustehen und an denen selbst staatliche Gewalt endet, definierte. Angesichts der Allgewalt Gottes und des von ihm durch seinen Stellvertreter regierten Staates konnte der einzelne als selbständige Einheit mit Rechten außerhalb der religiösen und staatlichen Kompetenzen nicht ins Blickfeld treten; es wäre dies wohl tatsächlich als so etwas wie „Vielgötterei“ erschienen. Die Interessen des Individuums müssen im Interesse des ganzen Staates aufgehen. Sein Leben, seine Macht und sein Glück dürfen nicht für sich selbst stehen; es muß lernen einzusehen, daß es keine eigene Existenz außerhalb der Existenz des Staates hat. Ein Muslim gewinnt so seine Identität erst als ein Mitglied der islamischen "umma“, und indem er sich für deren Erhaltung, Prosperität und Erweiterung einsetzt ${ }^{7}$.

\section{Die historische Entwicklung des islamischen Staates}

Solange die Führung des islamischen Staates religiösen Grundsätzen genügte, mag eine Identifizierung zwischen dem einzelnen und dem Staatswesen möglich und fruchtbar gewesen sein. Der hohe Anspruch ließ sich indessen nicht lange aufrechthalten, und rasch entwickelten sich Staatsideal und realpolitische Wirklichkeit auseinander. Bereits die umaiyadischen Kalifen (661-750) gaben dem islamischen Staatswesen zunehmend machtpolitische Züge. Als dann die Abbasiden das Umaiyadenhaus stürzten (750) und das Kalifat an sich rissen, suchten sie ihm zwar zunächst bewußt eine religiöse Prägung zu geben, doch traten die Spannungen zwischen der sich unter dem Einfluß der Tradition der persischen Großkönige zunehmend realpolitisch gerierenden Herrscherklasse und der von ihnen eingesetzten Administration einerseits und den Untertanen andererseits hervor. Die ehemals ideale einheitliche Gemeinde teilte sich in zwei Klassen. Zudem ging auch die geographische Einheit des Staates verloren: Ein Umaiyade floh nach Spanien und errichtete dort ein Gegenkalifat, das sich als dauerhaft erwies (bis ins 11. Jahrhundert).

\footnotetext{
6 S. J. H. Kramers: L'Islam et la démocratie. In: Orientalia Nederlandica. A Volume of Oriental

7 A. K. Faizuddin Ahmed: State and Individual in Islam. In: The Islamic Literature, Bd. 7, Nr. 5 (Mai
} 1955), S. 13-20. 
Später (seit 969) errichteten die Fatimiden in Kairo sogar ein schiitisches Kalifat.

Auch überall sonst in der islamischen Welt entstanden Fürstentümer, die faktisch selbständig waren, obwohl sie eine formale Oberhoheit des Kalifen anerkannten. Und seit dem 10. Jahrhundert gerieten die abbasidischen Kalifen in ihrer eigenen Hauptstadt Bagdad in offene Abhängigkeit von fremden Söldnerführern, die sie früher zu ihrem Schutze herbeigerufen hatten und die die Kalifen nach eigenen Interessen ein- und absetzten. Von der Vorstellung des universalen Reiches, das der Kalif als Mittelsmann Gottes unter dessen Gesetz regieren sollte, und der hohen Konzeption des islamischen Staates als der zeitlichen Manifestation des Wirkens Gottes, an dem mitzuarbeiten der einzelne seinen Sinn, seinen Wert und in dem er seine Rechtstellung erhalten hatte, blieb kaum etwas übrig.

Die Folge war, was als "orientalischer Despotismus" seit den Anfängen der Geschichten des Orients immer wieder als typisch orientalische Herrschaftsform bezeichnet worden ist. Nachdem der Sinn des Staatswesens entleert ist, bleibt doch der Zentralismus und Absolutismus des Herrschers und seiner Administration, die zur Zeit des intakten Kalifates religiös fundiert waren, bestehen. Das Fehlen jeglicher Institutionen unabhängig von der zentralen Macht, die in der Lage gewesen wären, die Gewalt des Herrschers zu beschränken, zu kontrollieren oder dieser einen organisierten Willen der Untertanen entgegenzusetzen, gibt jener, die nunmehr keine andere faktische Legitimation kennt als die eigene Stärke, die totale Verfügungsgewalt über den Staat und die Untertanen. Diese werden zum Spielball dynastischer Interessen. Auf Seiten der Untertanen schließlich führt diese Entwicklung zu einem weitgehenden politischen Quietismus. Es interessiert sie immer weniger, wer das Staatswesen verwaltet und auf welchem moralischen Niveau der Kalif und die jeweilige Regierung stehen, solange nur die wichtigsten der Bestimmungen des islamischen Rechts (sharî’a) eingehalten werden, und es ihnen möglich ist, ihr irdisches Dasein entsprechend dem göttlichen Gesetz einzurichten. Es ist ein oft zitiertes Dictum, das besagt: „Wenn der Kalif (oder der jeweilige Herrscher) gerecht ist, so ist sein Anteil die Belohnung und der deine dankbar zu sein; ist er ungerecht, so ist sein Anteil die Sünde, und der deine geduldig zu sein." 8

\section{„Menschenrechte“ und islamischer Modernismus}

In der Flut neuerer islamischer Literatur über das Problem des „islamischen Staates", d. h. über die Frage, ob und in welchem Umfang in unserer Zeit der Islam zur Grundlage eines modernen Staatswesens gemacht werden könne ${ }^{9}$, wird immer wieder der "demokratische" Charakter eines solchen hervorgehoben. In dem Versuch, apologetisch die Zeitgemäßheit der rechtlichen, institutionalen, gesellschaftlichen und ideologischen Aspekte des islamischen Gesamtsystems zu „er-

\footnotetext{
8 Ibn Abd Rabbihî: Kitâb al-iqd al-farîd, Kario, Bd. 1, 1940, S. 10. Zum Niedergang der islamischen Staatsauffassung, dargestellt an der Entwicklung der Kalifatstheorie, s. Enzyklopädie des Islams, 1. Aufl., s. v. "khalîfac, II, $945 \mathrm{ff}$; ; T. W. Arnold: The Caliphate, 2. Aufl., London 1965; G. E. von Grunebaum: Der Islam im Mittelalter, Zürich 1963, S. 169-204; R. Levy: The Social Structure of Islam. Being the second edition of the Sociology of Islam, Cambridge 1962, S. 277-299.

9 S. z. B. Niaz Ahmed Zikria: Les principes de l'Islam et la démocratie, Paris 1958; Raihan Sharif: An Islamic State - a Realizable Idea. In: The Islamic Review, Januat 1954; Zaman, Badiuz: Islamic State A Realizable Idea. In: The Islamic Review, Bd. 7, Nr. 7, Juli 1955, S. 5-18; Saghir, Hassan Masumi: A Realizable Idea. In: The Islamic Review, Bd. 7, Nr. 7, Juli 1955, S. 5-18; Saghir, Hassan Masumi:
Concept of Islamic State as I Understand it. In: ibid., September 1955. Vgl. B. Lewis: The Concept of Concept of Islamic State as I Understand it. In: ibid., September 1955. Vgl. B. Lewis: The Concept of
an Islamic Republic. In: Die Welt des Islam, Bd. 4, Nr. 1, Leiden 1955; H. Reiners: Die klassische islamische Staatsidee, ihre moderne Interpretation und ihre Verwirklichung in den Verfassungsordnungen muslimischer Staaten, Diss., Münster 1968.
} 
weisen“, werden dabei mit Nachdruck die "Menschenrechte"10 als der islamischen Lehre immanent apostrophiert. Genannt werden insbesondere die Gleichheit aller Menschen ohne Unterschiede von Rasse, Hautfar've oder ihrer religiösen Úberzeugung; die Glaubens- und Meinungsfreiheit; das Recht auf Freiheit der Person; das Recht auf Eigentum; sowie das Recht auf Erhaltung und Respekt des Lebens und der Ehre. Angesichts des oben ausgeführten bedarf dies der Erläuterung und der kritischen Untersuchung.

Der Kern des Islam läßt sich - soweit er den einzelnen Gläubigen betrifft - als einen Glaubensakt zusammenfassen, der aus zwei Elementen besteht, die im Glaubensbekenntnis zusammengefaßt sind: Unterwerfung unter den einen Gott und die Bejahung der prophetischen Mission, die dieser Mohammed als dem letzten einer Reihe von Vorgängern in der Prophetenmission anvertraut hat. Zwischen Allah und dem Gläubigen besteht eine unmittelbare Verbindung, die in der für alle verbindlichen Offenbarung des Korans, aus dem wiederum die allgemein gültigen Rechte und Pflichten in Staat und Gesellschaft abgeleitet sind, einerseits und in dem von allen unmittelbar vollzogenen Gebet andererseits besteht. Eine Zwischenschaltung eines Mittlerstands etwa in Form eines Priestertums, einer Kirche oder eines Standes privilegierter Rechtsgelehrter kennt der Islam nicht. Das bedeutet, daß ein Muslim dem anderen religiös völlig identisch ist. Indem der Islam zugleich ein einheitliches soziales Ganzes bildet, verwirklicht er völlige Gleichheit, die auf allen Ebenen des Lebens, die von ihm bestimmt werden, wirksam ist. Der Herrscher selbst, der nach der klassischen Theorie eigentlich aus einer Wahl hervorgegangen sein muß, unterliegt dem Religionsgesetz wie jeder andere Muslim; er ist nur "primus inter pares“. Dieser Sachverhalt hat zahlreiche moderne Apologeten bewogen, vom Islam als einer „demolrratischen Religion“ zu sprechen.

Die Freiheit des Glaubens und der Meinung wird von ihnen in der Stellung von Christen und Juden innerhalb des islamischen Imperiums als gegeben angesehen. Vom Beginn der islamischen Ausbreitung an stellte sich das Problem des Status der zahlreichen in den eroberten Gebieten lebenden Andersgläubigen, die sich nicht zu der neuen Religion bekehren wollten. Sie gewaltsam zu bekehren, hatte der Prophet selbst zurückgewiesen; sie andererseits voll an der politischen Gewalt partizipieren zu lassen, verbot der Charakter des islamischen Staates selbst. Das Problem wurde so gelöst, daß die „Buchbesitzer" (ahl al-kitâb), d. h. die Angehörigen von Offenbarungsreligionen, deren Propheten von Mohammed als seine "Vorläufer" anerkannt wurden (es handelt sich um Christen, Juden und Zoroastrier), als „Schutzbefohlene" (ahl al-dhimma oder dhimmî11) einen quasi autonomen Status unter islamischer Oberhoheit erhielten; sie genossen so ein weithin freies religiöses und soziales Leben. Ihre Rechte hatten keinen politischen Charakter im Sinne einer verfassungsmäßigen rechtlichen Gleichstellung mit den Muslimen, lebten sie doch mehr neben als in der islamischen Gemeinde. Doch wurden ihnen zahlreiche Zugeständnisse gemacht, die dem „Menschenrecht" der Freiheit des Glaubens und der Meinung nahekommen. Als Gegenleistung waren ihnen eine Kopfsteuer (djizya) ${ }^{12}$ und die Annahme loyalen Verhaltens gegenüber dem Islam auferlegt.

\footnotetext{
$10 \mathrm{~S}$. E. Rabbath: La théorie des droits de l'homme dans le droit musulman. In: Revue Internationale de Droit Comparé, 1959; N. J. Coulson: The State and the Individual in Islamic Law. In: International and Comparative Law Quarterly, Jan. 1957; Henry Siegmann: The State and the Individual in Sunni Islam. In: The Muslim World, Bd. 54/1, Jan. 1964.

11 S. Enzyklopädie des Islams, 2. Aufl., s. v. „dhimmî*.

12 Ibid., s. v. „djizya".
} 
Daneben werden von den Modernisten noch allgemeinere Argumente ins Feld geführt, die beweisen sollen, daß die „Menschenrechte“ im Islam stets ihren festen Platz gehabt hätten. So sei es die Mission des Islam von Anfang an gewesen, die Ungerechtigkeit zu beseitigen, das Böse zu unterdrücken und den Sieg der Gerechtigkeit herbeizuführen - der Gerechtigkeit unter all ihren Aspekten: zum Wohle des Individuums wie zum Wohle aller Gemeinschaften ohne Unterscheidung der Rassen oder Religionen. Recht (hakk) und Gerechtigkeit (adl) seien Leitmotive koranischer Sprache, und die "Anleitung zu dem, was statthaft ist, und das Abhalten von dem, was verboten ist" (al-amr bil-ma'rûf wan-nahy ani 'l-munkar), stelle die fundamentale Norm des religiösen Rechts und der Moral des Islam dar.

Gegen die islamischen Modernisten und Apologeten können indessen folgende Einwände vorgebracht werden:

Erstens: Bezugssystem und Rahmen der „Rechte“ bleibt die islamische Religion; innerhalb des islamischen Reiches haben Nicht-Muslime nur die Stellung einer geduldeten Minderheit, die neben der „umma“ lebt. Für die Muslime waren sie Menschen zweiter Klasse. Darüber hinaus ist der Anwendungsbereich des Status als „dhimmî" nur auf die „ahl al-kitâb“ beschränkt: Die Bekenner anderer Religionen standen vor der Alternative Bekehrung oder Tod. Die absolute Úberordnung des islamischen Rahmens über den Bereich der "Menschenrechte“ kommt schließlich in der Scheidung zwischen "politischen“ und „zivilen“ Rechten zum Ausdruck: Erstere waren allein Muslimen, letztere allen zugestanden.

Zweitens: Die skizzierte Entwicklung der islamischen Staatskonzeption vom Ideal einer Art Gottesstaates zur nackten Despotie und die totale Ohnmacht des einzelnen in ihr zeigen, daß die rechtliche Stellung des einzelnen im Staat völlig ungesichert war, daß jener gegenüber dem Staat und der Obrigkeit keineswegs über jenen unverletzlichen Bereich verfügte, den die "Menschenrechte“ dem Individuum zusichern.

Drittens: Die religiöse Gleichheit der Muslime als Glaubensbrüder, die durch die Unmittelbarkeit der Beziehung eines jeden einzelnen zu Gott gewährleistet ist, hat die Herausbildung einer Gesellschaft mit abgestuften und differenzierten Klassen und Privilegien nicht verhindert. Auch wenn Durchlässigkeit und Mobilität der islamischen Gesellschaft größer waren als im christlichen Bereich, ist doch de facto eine hierarchische Struktur gegeben gewesen.

Viertens: Die Frau war dem Mann rechtlich untergeordnet.

Fünftens: Auch die Glaubensfreiheit war nicht allen zustehendes Recht, sondern religiös begrenzt: Zwar konnte ein Christ oder Jude seinem Glauben treu bleiben oder zum Islam konvertieren. Umgekehrt aber war es dem Muslim bei Todesstrafe verwehrt, seinen Glauben zu verlassen.

An keiner Stelle innerhalb der staatstheoretischen, philosophischen oder juristischen Literatur vor dem Zeitpunkt, da das moderne Europa begann auf den Islam einzuwirken, hat sich eine Stimme erhoben, die Rechte des Individuums zu fordern. Zwar hat es zahlreiche Volksbewegungen, politische Umstürze, soziale Revolutionen gegeben, die die Vitalität der islamischen Gesellschaft trotz der von oben auferlegten Immobilität bezeugen. Wenn aber dennoch eine Doktrin der individuellen Freiheiten im Islam nie geschaffen wurde, so wohl deshalb, weil die Notwendigkeit, dies zu tun, nicht empfunden wurde. An der Stelle einer geschlossenen Doktrin hat das islamische Recht versucht, dem Menschen ein Instrumentarium von praktischen Regeln, Konzepten, Garantien bereitzustellen, und so einen Rah- 
men des Gemeinschaftslebens zu schaffen, innerhalb dessen der einzelne sein Leben einrichten konnte.

Die deutliche Spannung zwischen der absoluten Unterordnung des Muslims unter dem Willen Gottes und seiner vollständigen Einordnung in die Interessen des ganzen, der "umma“, des islamischen Staates als der zeitlichen Manifestation des Wirkens Gottes auf der einen und dem Versuch der Erarbeitung eines passablen Modus vivendi für den einzelnen auf der anderen Seite ist ein Grundzug des Islam als ganzem auch außerhalb des im Rahmen unseres Themas untersuchten politischsozialen Bereichs. Dem Islam wohnt ein Element nüchterner Vernünftigkeit inne, das auf eine humane Durchführung seiner Vorschriften hinwirkt. „Allah wünscht, es euch leicht zu machen; und er wünscht nicht, es euch schwer zu machen "13, ist einer der Leitsätze islamischer Ethik. Der Islam ist in hohem Grade human, in dem Sinne, daß er den Menschen nimmt, wie er ist. Er ist aber nicht humanistisch, in dem Sinne, daß er sich um die möglichst reiche und möglichst volle Entfaltung und Entwicklung der im Menschen liegenden Möglichkeiten bekümmerte und daß ihm die Menschenformung als die erste und vornehmste Aufgabe der Kultur erschiene und der Mensch selbst als sein eigenes und gleichsam absolutes Maß ${ }^{14}$.

\section{Die „Menschenrechte“ in der heutigen Welt des Islam}

Die ägyptische Expedition Napoleons (1798) wird gemeinhin als der Zeitpunkt gesetzt, an welchem die Unterlegenheit der islamischen Welt auf allen Gebieten endgültig sichtbar wurde, und ihre rasche politische Unterwerfung unter Mächte des Westens ihren Anfang nimmt. Für den Muslim waren damit nicht nur der Rahmen seiner politischen Existenz, sondern die Grundlagen seines gesamten Daseins in Frage gestellt. Gemäß seinem Staatsverständnis war sein Staat ja nicht eine autonome Größe, sondern in ihm war Gott selbst unmittelbar wirksam; d. h. der Zustand des Staates, sein Gedeihen und sein internationales Ansehen waren für ihn zugleich Aussagen über Gott, sein Wirken, ja seine Existenz. Wenn die politische Stellung der Gemeinde Gottes unaufhaltsam sich zu verschlechtern schien, wie war es da mit Gott gestellt: Hatte er sich vor der Gemeinde zurückgezogen? Hatten die Gläubigen die Mittel, mit denen er sie gelenkt hatte, das göttliche Gesetz, verfälscht oder nicht mehr richtig angewandt? Was galt es also zu tun, um die politische Ordnung der islamischen Welt wieder auf jene Höhe zu bringen, die von dem Muslim als natürlich und angemessen empfunden wurde und auf der sein Selbstverständnis als Muslim wieder „in Ordnung“ war? Die politische Krise war also unabtrennbar zugleich eine geistig-kulturelle und diese wiederum nicht $\mathrm{zu}$ lösen ohne eine neue tragfähige politische Ordnung.

Gegen Ende des 19. Jahrhunderts glaubten die politischen Führungsschichten des Nahen Ostens, den Schlüssel der Úberlegenheit des Westens gefunden zu haben. Es schienen die westlichen Staatsideen und politischen Institutionen zu sein, die diesem eine solche Dynamik verliehen; sie zu übernehmen, mußte bedeuten, die islamische Welt aus Krise und Stagnation herauszuführen und ihr neue Vitalität zu verleihen. Insbesondere von der Hinwendung zum Nationalismus und den kon-

13 Koran II, 181.

14 S. G. E. von Grunebaum: Der Islam im Mittelalter, Zürich und Stuttgart, 1963, S. 295 f. 
stitutionellen und demokratischen Instrumenten und Institutionen erhoffte man die umfassende politische Erneuerung.

Diese Entwicklung aber bedeutete zunächst einen mehr oder minder offenen Bruch mit der traditionellen islamischen Ordnung und die Aufgabe des theokratischen islamischen Staatsgedankens und des religiös fundierten gesellschaftlichen Systems. Dies zugunsten einer Ordnung, die auf Territorium, Sprache, Abkunft aufbaute und in "dem Menschen“ das gestaltende Grundelement eines Gemeinwesens erblickte, das seine Legitimation nicht in Gott als dem einzigen Souverän erfuhr, sondern seine Souveränität in sich selbst barg. Anstelle des Herrschers, der autokratische Gewalt als Statthalter Gottes auf Erden und Hüter des göttlichen Gesetzes ausübte, traten nunmehr konstitutionelle Elemente wie Verfassungen, Parlamente und Parteien.

Auf dem Territorium des während des Ersten Weltkriegs zerfallenen Osmanischen Reiches entstanden eine Reihe Nationalstaaten, die sich territorial, rassisch, historisch, ideologisch, wirtschaftlich und politisch gegeneinander abgrenzten. Hatte sich das Osmanische Reich zumindest formal noch als politischer Repräsentant der "umma" verstanden, so erschien diese nun politisch zersplittert und allenfalls auf der Basis des religiösen Bekenntnisses zum Islam und gelegentlicher gesamtislamischer Konferenzen fortbestehend.

Dieser Prozeß der Modernisierung schuf auch für die „Menschenrechte“ im islamischen Bereich einen neuen Rahmen. Bereits 1839 hatte der osmanische Sultan Mahmud II. unter dem Einfluß französischer und belgischer Vorbilder im Rahmen seiner allgemeinen Reformmaßnahmen, die unter dem Namen "hatt-i scherîf“ (großherrliches Handschreiben von Gülhane) in die Geschichte eingegangen sind, einen Grundrechtskatalog erlassen. Von besonderem Interesse war hier neben anderem die praktische Gleichstellung der islamischen und nicht-islamischen Untertanen des Sultans, insbesondere durch die Bestimmung der Gleichheit aller bei der Heranziehung zum Militärdienst. War die Verteidigung des islamischen Staates bislang ein natürliches Prärogativ der Muslime selbst, so bedeutete die Maßnahme einen deutlichen Schritt in Richtung auf eine Entflechtung des Staates aus seinem religiösen Zusammenhang. Nach der Verabschiedung von regelrechten Verfassungen im Osmanischen Reich (1876) und Persien (1906/7) sind Verfassungen nach dem Ende des Ersten Weltkriegs in nahezu allen Nachfolgestaaten des Osmanischen Reiches verkündet worden. In ihnen sind durchweg die wichtigsten Grundrechte verankert. Unter anderen werden genannt: die Gleichheit aller Bürger vor dem Gesetz, die Unverletzbarkeit der persönlichen Freiheit, die Unverletzbarkeit der Wohnung, die Freiheit der Religionsausübung, die Meinungsfreiheit, das Vereinigungsrecht.

Die Frage, die sich damit stellt, ist jedoch, ob in diesen Entwicklungen tatsächlich eine vollständige Angleichung der politischen Systeme, ob ein tiefgreifender Wandel der für den Islam so charakteristischen Beziehungen von Religion, Staat und Individuum stattgefunden hat, oder nicht vielmehr klassische islamische Konzeptionen nachwirken. Nur ein paar Hinweise seien hier gegeben:

1. Die in die Verfassungen eingeführten Grundrechte bleiben im allgemeinen nur unvollkommen abgesichert. Zieht man als Beispiel die ägyptische Verfassung vom 25. März 1964 heran $^{15}$, so stellt man fest, daß ein echter Schutz der Grund-

15 Vgl. R. Büren: Nassers Agypten als arabisches Verfassungsmodell, Opladen 1972, S. 33 f. 
rechte gegenüber der staatlichen Gewalt nicht wirklich gegeben ist. Etwa die Hälfte der Grundrechte und staatlich garantierten Einrichtungen ist nach dem Wortlaut der Verfassung der Verfügung der staatlichen Gesetzgeber unterstellt. Diese sind die Nationalversammlung sowie der Präsident der Republik. Der staatliche Gesetzgeber, ob Nationalversammlung oder Präsident, ist an keinerlei Rechte oder Grundrechte gebunden. Ausdrückliche Sicherungen wie das Verbot der individuellen Durchbrechung der Grundrechte oder die Unantastbarkeit des Wesensgehalts waren der genannten Verfassung unbekannt. Auch die Grundrechte, die ihrem Wortlaut nach nicht zur Verfügung des staatlichen Gesetzgebers stehen, genießen keinen hinreichenden Schutz. Da sie kein unmittelbar anwendbares Recht darstellen - eine Bestimmung wie Artikel 1 III Grundgesetz fehlt -, entfalten sie lediglich eine programmatisch-direktive Wirkung.

2. Es gibt Indizien dafür, daß der Absolutheitsanspruch des islamischen Staates gegenüber dem Individuum auch in modernen Nationalstaaten fortbesteht: Die unzureichende Absicherung der Grundrechte entspricht der Stellung des einzelnen in der "umma" des arabisch-islamischen Staates. Zum anderen ist auf die Vorrangstellung des Staatspräsidenten im Rahmen der Verfassung hinzuweisen, die weder durch das Parlament noch die Judikative vollkommen kontrolliert wird. Dieser Sachverhalt weist Züge der Stellung des Kalifen im klassischen islamischen Staat auf, in welchem auch neben diesem als "vicarius dei“ keine legitime selbständige Gewalt bestehen konnte. Der autoritäre Charakter nahezu aller nahöstlichen Regime und das Auftreten charismatischer Führer zwischen Marokko und Pakistan wären so letztlich aus einer vielhundertjährigen islamischen Tradition zu verstehen. In diesem Zusammenhang ist auch die Rolle der Parteien interessant. Es ist bezeichnend, daß in zahlreichen islamischen Scaaten - genannt seien Algerien, Libyen, Tunesien, Ägypten und der Sudan - Einheitsparteien bestehen, was einer Quasi-Monopolisierung der Gestaltung des politischen Lebens durch diese Organisationen gleichkommt. Wo es eine Einheitspartei nicht gibt, werden die aktiven Kräfte von einer dominierenden Partei im Rahmen einer Art "Nationaler Front" wie etwa in Syrien oder im Iraq unter der Baath-Partei geführt.

Die Einheitspartei ist nicht Partei im westlichen Sinne: Sie ist unmittelbarer Ausdruck des Selbstverständnisses der Gesamtheit der politischen und gesellschaftlichen Kräfte. Die Vorstellungen von der weiteren Entwicklung von Staat und Gesellschaft sind allen auf so selbstverständliche Weise gemeinsam, wie dies in der von Gott gelenkten „umma“ durch die islamische Geschichte theoretisch der Fall gewesen ist. Die Partei erscheint mithin als ein moderner Ausdruck der klassischen arabisch-islamischen Konsensualgemeinschaft. Ihre Bedeutung besteht vor allem darin, den Konsens (idschma') dieser Gemeinschaft in die politische Praxis umzusetzen und $\mathrm{zu}$ organisieren ${ }^{16}$. Als eine Manifestation der Konsensualgemeinschaft kann es nur eine politische Organisation in einem Staat geben - andere Parteien sind subjektive Abweichungen vom objektiv notwendigen Gang der Dinge. In diesem Sinne betrachtet das libysche Parteigesetz vom 30. 5. 1972 in Artikel zwei parteipolitische Aktivitäten als einen Akt von Verrat gegen das Volk ${ }^{17}$.

16 Vgl. R. Büren: Die Arabische Sozialistische Union, Opladen 1970, S. 226. Text in Verfassungstexte "Aegypten ${ }^{\alpha}$, Beilage zu VRU 1972, Heft 2 der neuen Verfassung.

17 "Summary of World Broadcasts, Part 4. The Middle East and Africa. Published by the monitoring service of the British Broadcasting Corporation, 4004/A/1, 2. 6. 1973. 
Dies macht deutlich, daß trotz der Übernahme moderner staatspolitischer Elemente ein Wandel überkommener Auffassungen nur teilweise stattgefunden hat. Noch immer ist der Staat in seiner politischen Sinngebung dem Wert des Einzellebens übergeordnet. Und trotz der Bekundung der Rechte des Menschen bleibt dieser als einzelner politisch unbeachtet und gegenüber dem Ganzen von untergeordneter Bedeutung. Oder wie es der libysche Ministerpräsident Jallud ausgedrückt hat: Gegenüber dem kollektiven Konsens der „umma“ habe der einzelne keine Bedeutung, sondern könne allenfalls diesen Konsens verfälschen ${ }^{18}$.

3. Trotz der ausdrücklichen Fixierung des Gleichheitsprinzips in den Verfassungen gibt es zahlreiche, aus traditionellem islamischem Recht stammende Vorbehalte gegen dessen Verwirklichung. Bekannt ist die de facto bestehende Abstufung der Geschlechter: Trotz lebhafter Diskussion der Frauenfrage und der Aktivität von Frauenverbänden bleibt die Frau in ihrer rechtlichen, gesellschaftlichen und politischen Stellung dem Mann nachgeordnet. Der libysche Staatspräsident al-Qadhafi hat anläßlich einer Diskussion mit ägyptischen Frauenverbänden in Kairo (Juli 1973) die körperlichen Eigenheiten beschrieben, die eine spezifische Rolle der Frau in der Gesellschaft determinieren. Und die neue ägyptische Verfassung vom September 1971 sieht in Artikel elf zwar grundsätzlich die Gleichstellung von Mann und Frau im politischen, sozialen, kulturellen und wirtschaftlichen Leben vor, schränkt allerdings ein: „... ohne die Normen der „sharî’a“ zu verletzen ${ }^{19}$.“

Ungleichheiten entstehen auch aus der religiösen und rassischen Zugehörigkeit: In zahlreichen nahöstlichen Staaten gibt es mehr oder minder starke religiöse (etwa christliche oder jüdische) oder rassische (etwa kurdische) Minderheiten. Mag auch ihr zivilrechtlicher Status mit Einschränkungen demjenigen der arabischmuslimischen Mehrheit ähnlich sein, so sind sie doch in ihren politischen Rechten eingeschränkt, unterprivilegiert und als Gruppe unterrepräsentiert. Ohne auf Einzelheiten einzugehen, sei hier nur an die Kopten in Ägypten und die Kurden im Iraq erinnert.

4. Die - wenn auch in veränderter Form - fortbestehende Zusammengehörigkeit von Religion und politisch-sozialem System kommt in der Tatsache zum Ausdruck, daß der Islam als die Grundlage der Gesetzgebung oder als „Staatsreligion“ in fast allen arabischen Staaten (und Pakistan) ${ }^{20}$ anerkannt wird. Dies gilt fraglos für Saudi Arabien, dessen ganzes Rechtssystem noch auf dem religiösen Gesetz (sharî’a) aufgebaut ist und das deshalb keiner „Verfassung“ bedarf. Das gilt auch für die Emirate der Arabischen Halbinsel, die erst in jüngerer und jüngster Zeit im Zuge der Gewinnung der Unabhängigkeit und politischen Selbständigkeit von Großbritannien verfassungsmäßige Organe eingeführt oder sich ordentliche Verfassungen gegeben haben. Besonders signifikant indessen ist die Betonung der Stellung des Islam im Rahmen des Staatsgefüges in den sogenannten "progressiven" arabischen Staaten. Algerien z. B. hat den Islam zur Staatsreligion erhoben ${ }^{21}$. Die ägyptische Verfassung geht weiter; dort heißt es in Abschnitt 1, Artikel 2: „Der

18 Summary of World Broadcasts, ME/4125/A/10, 23. 10. 1972. Vgl. Ahmad Nur: Islamic Democracy versus Modern Western Democracy. In: The Islamic Literature, Bd. 9, Nr. 1/2, Januar-Februar 1957; Muhammad Abdullah al-Araby: The Concept of State in Islam: Western and Islamic Concepts of State. In: The Islamic Review, Juli-August 1959.

19 Oriente Moderno, Rom, Nr. 51, 1971, S. 687; Verfassungstexte „Aegypten ${ }^{\alpha}$ (Anm. 16).

20 Verfassungstexte "Pakistan “, Beilage zu VRU 1974, Heft 2. Nicht mehr dagegen in Bangladesh, vgl. Verfassungstexte „Bangladesh“, Belage zu VRU 1973, Heft 4.

21 S. Oriente Moderno, Nr. 43, 1963, S. 651-656. 
Islam ist die Religion des Staates; Arabisch ist seine offizielle Sprache. Die Prinzipien der islamischen "sharı̀'a" sind eine Hauptquelle der Gesetzgebung22."

Indem das islamische Recht in den Mittelpunkt der staatlichen Existenz gerückt wird, ist angezeigt, daß auch heute ein islamischer Staat seine Legitimation aus dem Wirken Gottes in ihm ableitet. Und dies bedeutet gleichzeitig, daß auch die Stellung des Menschen zum Teil noch von jenen Vorstellungen geprägt ist, die als für den Islam konstituierend herausgearbeitet wurden. Dort also wo in islamischen Verfassungen nach westlichem Vorbild Grundrechte des Menschen verankert werden, sind sie zwar mehr als nur Zugeständnisse an einen überlegen erscheinenden kulturellen und politischen Raum; sie entspringen durchaus dem echt humanen Zug, der dem Islam stets innewohnte. Sie bedeuten aber noch nicht ein klares Bekenntnis zu einer Welt des Menschen als dem Ausgangspunkt und dem Ziel der irdischen Ordnung. Sondern letztlich handelt auch jetzt noch der Mensch ad maioren dei gloriam, d. h. islamisch gesprochen: zur Erfüllung des Willens Gottes auf Erden. Insofern liegt die Priorität nicht bei ihm als Träger unabdingbarer Rechte, sondern bei der "sharî'a“ als der letzten politischen und gesellschaftlichen Richtschnur.

In der Politik im Nahen Osten ist gegenwärtig ein Prozeß der "Islamisierung“ erkennbar. Das bedeutet, daß die Außenpolitik, die Innen- und Gesellschaftspolitik arabischer Staaten ganz deutlich wieder stärker auf islamischem Fundament gründen. Zahlreich sind die Indizien; genannt sei hier nur die verstärkte Rolle des islamischen Staates Saudi Arabien auf gesamtarabischer Bühne. Den radikalsten Versuch, den Islam in vollem Umfang wieder zur Grundlage der gesamten politischen Existenz zu machen, stellt die libysche „Kulturrevolution“ al-Qadhafis dar. Auf eine Formel gebracht ist ihr Ziel, Gott und seine Gemeinde wieder in jene unmittelbare Beziehung zu bringen, die Qadhafi als die Bedingung der großen Erfolge, der politischen Stärke und gesellschaftlichen Kohärenz der ersten Jahrhunderte des Islam sieht. Dazu sei es notwendig, das islamische Recht wieder zur Anwendung zu bringen und die „importierten“ westlichen Gesetze zu eliminieren ${ }^{23}$.

Die libyschen Maßnahmen können nicht als typisch für die Situation in der arabisch-islamischen Welt gewertet werden. Gleichwohl sind sie vielleicht symptomatisch für gewisse Entwicklungen, die nach einer langen Phase der Adaptation westlicher politischer und gesellschaftlicher Modelle wieder auf stärkere Abgrenzung zielen und nach einer Phase politischer und kultureller Dominanz von außen die Eigenkräfte des islamischen Raumes wieder zu aktivieren beginnen.

Bedeutet diese Entwicklung einen Rückschritt ins Mittelalter und mit Bezug auf die Menschenrechte, daß der Islam einen zentralen Wert der modernen Welt nicht oder nur oberflächlich anerkennt? Wohl kaum! Die „Menschenrechte“ haben nur einen anderen Stellenwert; der moderne Muslim bestreitet nicht, daß Aufstellung und Wahrung der Rechte des Menschen auf Erden eine zentrale Aufgabe des Menschen darstellen; nur bestreitet er, daß sie dem Menschen als solchem zukommen. Nach seiner Vorstellung kommen sie ihm zu, weil der Mensch der Träger der göttlichen Ordnung auf Erden ist; und insofern dieser an dieser Ordnung mitbaut, ist er gleich und frei und unverletzbar.

22 S. Oriente Moderno, Nr. 51, 1971, S. 686-689; Verfassungstexte „Aegypten “ (Anm. 16).

23 S. Summary of World Broadcasts, ME/4273/A/9-20, 17. 4. 1973; vgl. auch Bryde: Die Libysche Verfassungsproklamation vom 11. Dezember 1969, VRU 1970, S. 383 f. 
So haben die islamischen Staaten aus voller Überzeugung die „Menschenrechtserklärung der Vereinten Nationen" vom 10. Dez. 1948 unterzeichnet, wie sie aus voller Überzeugung in den Vereinten Nationen mitarbeiten. Denn die Aufgaben dieser internationalen Organisation im allgemeinen und der „Menschenrechtserklärung" im besonderen sind die Aufrichtung von Ordnung und Gerechtigkeit wie die Förderung des Guten und die Vernichtung des Bösen. Dies aber sind Aufgaben, mit denen sich der Muslim voll identifiziert und deren Vollbringung ein entscheidender Bestandteil seines politisch-religiösen Glaubens ist: Denn das Wirken Gottes in der Welt kann nur auf Ordnung und Gerechtigkeit zielen; diese können aber nur durch das Medium menschlicher Tätigkeit verwirklicht werden. Ist aber die Welt in Ordnung, in der Ordnung Gottes, dann ist auch sein Glauben „in Ordnung“. 
be changed to the Presidential form of government. No doubt, there is hardly any indication that the Indian "model" will be realized.

The development in Bangladesh proves that even the "best" constitution can not compensate for a deficit of efficiency, integrity, and rationality on Government's side.

\section{Human Rights in Islamic View}

\section{By Udo STEINBACH}

The conception of inalienable and prenational existing human rights, bestowed upon the individual person "by nature" has emerged from the specific framework of occidental culture. It presupposes the conviction of the individual person's autonomous existence. Islam has not been able to do this. According to Islamic reading, rooted in the indissoluble unity of religion and political existence in this world, the Islamic community, through its actions, bears witness to God's rule, who governs it directly through His law (shari'a). Thus, the Islamic state, being a manifestation of God in this world, is the superior order to the interest of which every individual must submit itself; the individual person has a value only in so far as he takes part in pursuing the goals and missions of the Islamic Community.

In view of the significance accorded to the state by Islam, individual rights existing "per se" were not conceived. Although Islamic lawyers have worked out a system of rules, according to which the individual person was able to create for himself a humane existence, the continuity of the national order was more important to them than preserving the individual person's fundamental rights. And since through the passage of Islamic history, particularly since the 10th century, the power of the state was converted into a tyranny by the rulers, the individual person increasingly became a subordinate, lacking will and rights.

Islamic modernists and apoligists have tried to show the existence of human rights, in the occidental meaning, in Islam and have tried to embody the equality of all people, freedom of religion, personal liberty, and the right to have life and honor respected. Especially the 19th and 20th century reform of the Islamic world in regard to the political principles of the Occident has made human rights a constituent of the constitutions of nearly every Islamic country. However, it is still obvious that elements of a traditional system are impeding a total recognition of human rights. In particular the embodiment in the constitutions of Islam being the state religion indicates that even today the priority of terrestrial order lies less with the individual person bearing inalienable rights than in the realization of God's will. 